NASA/TM-2003-212078

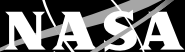

Damage Detection Using Holography and Interferometry

Arthur J. Decker

Glenn Research Center, Cleveland, Ohio 
Since its founding, NASA has been dedicated to the advancement of aeronautics and space science. The NASA Scientific and Technical Information (STI) Program Office plays a key part in helping NASA maintain this important role.

The NASA STI Program Office is operated by Langley Research Center, the Lead Center for NASA's scientific and technical information. The NASA STI Program Office provides access to the NASA STI Database, the largest collection of aeronautical and space science STI in the world. The Program Office is also NASA's institutional mechanism for disseminating the results of its research and development activities. These results are published by NASA in the NASA STI Report Series, which includes the following report types:

- $\quad$ TECHNICAL PUBLICATION. Reports of completed research or a major significant phase of research that present the results of NASA programs and include extensive data or theoretical analysis. Includes compilations of significant scientific and technical data and information deemed to be of continuing reference value. NASA's counterpart of peerreviewed formal professional papers but has less stringent limitations on manuscript length and extent of graphic presentations.

- TECHNICAL MEMORANDUM. Scientific and technical findings that are preliminary or of specialized interest, e.g., quick release reports, working papers, and bibliographies that contain minimal annotation. Does not contain extensive analysis.

- CONTRACTOR REPORT. Scientific and technical findings by NASA-sponsored contractors and grantees.
- CONFERENCE PUBLICATION. Collected papers from scientific and technical conferences, symposia, seminars, or other meetings sponsored or cosponsored by NASA.

- SPECIAL PUBLICATION. Scientific, technical, or historical information from NASA programs, projects, and missions, often concerned with subjects having substantial public interest.

- TECHNICAL TRANSLATION. Englishlanguage translations of foreign scientific and technical material pertinent to NASA's mission.

Specialized services that complement the STI Program Office's diverse offerings include creating custom thesauri, building customized databases, organizing and publishing research results ... even providing videos.

For more information about the NASA STI Program Office, see the following:

- Access the NASA STI Program Home Page at http://www.sti.nasa.gov

- E-mail your question via the Internet to help@sti.nasa.gov

- Fax your question to the NASA Access Help Desk at 301-621-0134

- Telephone the NASA Access Help Desk at 301-621-0390

- Write to:

NASA Access Help Desk

NASA Center for AeroSpace Information 7121 Standard Drive

Hanover, MD 21076 
NASA/TM-2003-212078

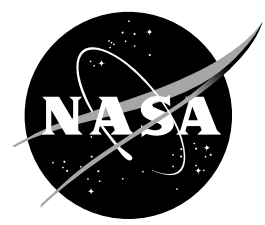

\section{Damage Detection Using Holography and Interferometry}

Arthur J. Decker

Glenn Research Center, Cleveland, Ohio

National Aeronautics and

Space Administration

Glenn Research Center 


\section{Acknowledgments}

I would like to thank Mr. Kenneth E. Weiland for providing invaluable technical assistance over the years and for preparing several figures.

Trade names or manufacturers' names are used in this report for identification only. This usage does not constitute an official endorsement, either expressed or implied, by the National Aeronautics and Space Administration.

Available from

NASA Center for Aerospace Information 7121 Standard Drive

Hanover, MD 21076
National Technical Information Service 5285 Port Royal Road Springfield, VA 22100 


\title{
DAMAGE DETECTION USING HOLOGRAPHY AND INTERFEROMETRY
}

\author{
Arthur J. Decker \\ National Aeronautics and Space Administration \\ Glenn Research Center \\ Cleveland, Ohio 44135
}

\section{INTRODUCTION}

Some major goals of structural testing are the detection of local damage and, more recently, the general evaluation of structural health. Detection of local damage calls to mind several specialized practices. The most direct is simple visual inspection for cracks or visual inspection perhaps enhanced by dye penetration. This simplest of approaches is augmented by a variety of visualization techniques. These include, ${ }^{1}$ for example, X-ray radiography; neutron radiography; ultrasonic techniques including $\mathrm{C}$-scan imaging; optical, electron and scanning probe microscopy; eddy currents; both optical and non-optical thermography and the interferometric methods to be discussed in this chapter.

The evaluation of the general health of structures depends on the detection and possibly sophisticated analysis of non-locally generated signatures. A very simple example to be discussed in this chapter is the measurement of the change in frequency of a resonant vibration mode of a structure that sometimes accompanies damage or cracking. A more sophisticated method is the detection of subtle changes in the vibration mode shape itself.

The minimum objective for all these technologies is to detect possibly injurious structural changes or structural damage. A more advanced objective is to quantify the magnitude of the structural changes or damage. 
In any case, the technologies often have little in common except for the objectives. This chapter will discuss specific optical techniques based on interferometry and holography. But the approach to be discussed for using neural networks to interpret interference patterns should be applicable to processing multiple channels of data from some of the other technologies.

\section{SURVEY OF HOLOGRAPHY AND INTERFEROMETRY FOR DAMAGE DETECTION}

\subsection{Principle of Operation}

Laser holography and interferometry have been used to detect structural damage nearly from the time of their discovery in the early 1960's. The approach to damage detection has not changed much since that time. An interference pattern or "fringe pattern" is created and monitored for the object of interest. Visual clues about changes in the structural integrity of the object are sought from variations in the interference pattern. Silver-halideemulsion holograms were used extensively for these inspections during the first twenty-five years of laser holography. Real-time, double-exposure and time-average holographic interferometry, as well as speckle interferometry, were used to generate the interference patterns. ${ }^{2}$ The important feature of the silver-halide emulsion was that it could store multiple holograms or speckle photographs of the surface of an object recorded at different times. ${ }^{3}$ The reconstructions from the different holographic snapshots of the time varying surface could then be accomplished at the same time. The reconstructed beams interfered, and non-uniformities or anomalies in the interference patterns were identified with damage. Holographic techniques are primarily sensitive to out-of-plane motions of the surface of an object, whereas speckle techniques are primarily sensitive to in-plane motions. Another optical method called moiré deflectometry could be used to detect local changes in the orientation of the surface of an object. ${ }^{4}$

The silver-halide emulsion has largely been replaced with electronic recording in the past fifteen years. ${ }^{5}$ Combination of the CCD camera with phase shifting interferometry offers most of the practical damage-inspection benefits of silver-halide-emulsion holography plus the ease of digital data acquisition and processing. The CCD camera has sufficient resolution to record image-plane holograms whose maximum spatial frequencies are set by low-pass filtering property of the camera lens and the accompanying 
pixel size. Unrestricted three-dimensional viewing still requires the silverhalide emulsion, but stereoscopic viewing can capture two perspectives from two electronic cameras. Electronic recordings of holograms and speckle patterns permit all the sophisticated tools of image processing and computing to be applied. But the user is still confronted with the challenges of detecting changes in fringe patterns and associating them with structural changes and damage.

\subsection{Classical Approaches}

Both early and more recent demonstrations of damage detection have often depended on rather dramatic structural failures such as severe cracks and de-bonding to generate spectacular local variations in the interference patterns. Figure 1 shows the rather obvious effect on a double-exposure fringe pattern of a de-bond defect in a flat-plate structure. Double exposures have been used dramatically to show separations in automobile tires, ${ }^{6}$ for instance. Various enhancements have been used to visualize less dramatic damage. One approach uses continuous phase shifting of the fringe pattern, and the accompanying local time variation in contrast, to call attention to a crack. Another approach is to visualize measured or computed patterns that map differences or derivatives of displacement rather than the surface displacements directly. ${ }^{7}$

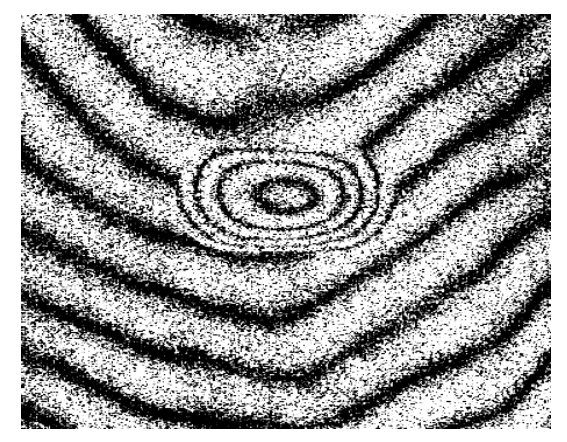

Figure 1. Defect shown by double-exposure fringe pattern

Figure 2 shows a fringe pattern from a time-average hologram of a simple mode of a cantilever, and the corresponding maps of the second derivatives of the interference phases for cracked and undamaged versions of that cantilever. The patterns in figure 2(b) and (c) were generated by an artificial neural network in response to the mode shape in figure 2(a). The second derivative mapped in figure 2(c) clearly shows the effect of a crack. 
The bending induced strain at the surface can, in fact, be calculated from the second spatial derivatives of displacement evaluated in the local tangent planes at the surface. ${ }^{8}$ The measured interference phase really maps the dot product of displacement and a sensitivity vector, thereby complicating the interpretation somewhat. But the sensitivity vector often varies slowly enough that the second derivatives approximately map bending induced strain. Regardless, the second derivatives of interference phase sometimes provide a way to visualize damage.

Optical inspections of solid surfaces for structural damage suffer from two major defects. First, inspections depend on human interpretation of visualizations and are observer dependent. A more serious defect is that optical patterns may not change dramatically in the presence of significant structural damage or changes.

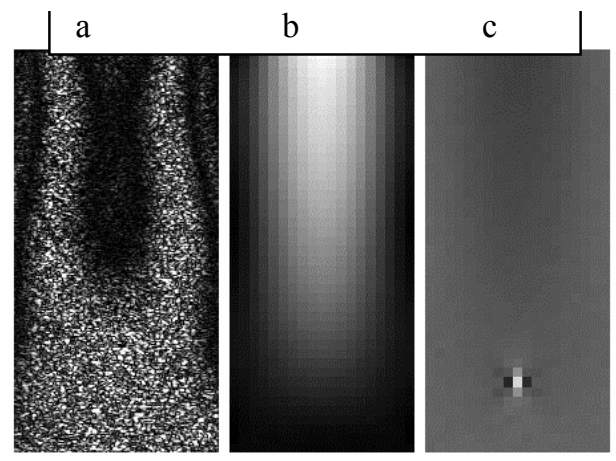

Figure 2. Fringe pattern (a) and second derivatives of interference phase from undamaged (b) and cracked (c) cantilevers.

As stated above, most demonstrations of holographic and interferometric techniques have depended on dramatic changes such as manifested by cracks and de-bonding. But structures can change substantially as measured by nonoptical methods without affecting the interference patterns recorded by using electronic holography. For example, a flat plate held on two sides by visegrips was vibrated in its fifth mode of bending, and electronic holography recorded the interference pattern of this mode (fig. 3). Each vise-grip was attached with four screws. The torque of one of these screws was adjusted back and forth from 0 to 70 inch-pounds, and the resonant frequency was recorded at 6 torque settings, with 10 measurements per setting.

Figure 4 shows that the resonant frequency of this vibration mode clearly changes with torque. But a visual examination of the associated vibration mode shapes shows no discernable variation. Two of these patterns are shown in figure 5. In other cases, mode shapes change when frequencies do not. 


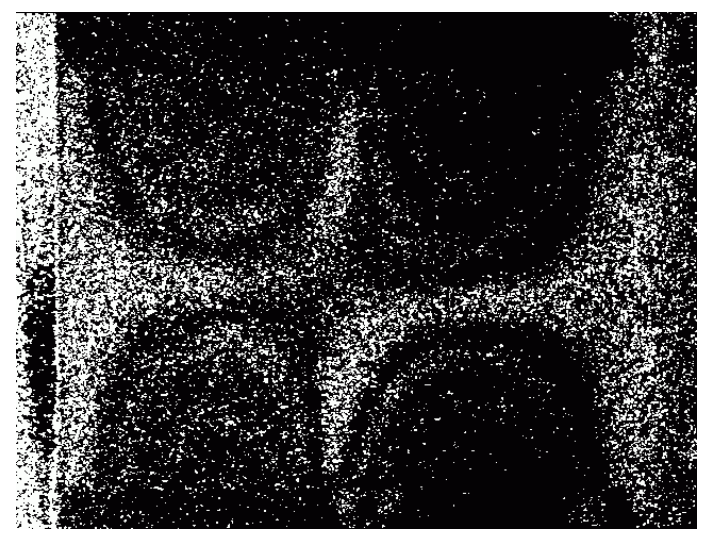

Figure 3. Fifth vibration mode of optical strain-gauge plate

Simultaneous Comparisons Between Groups

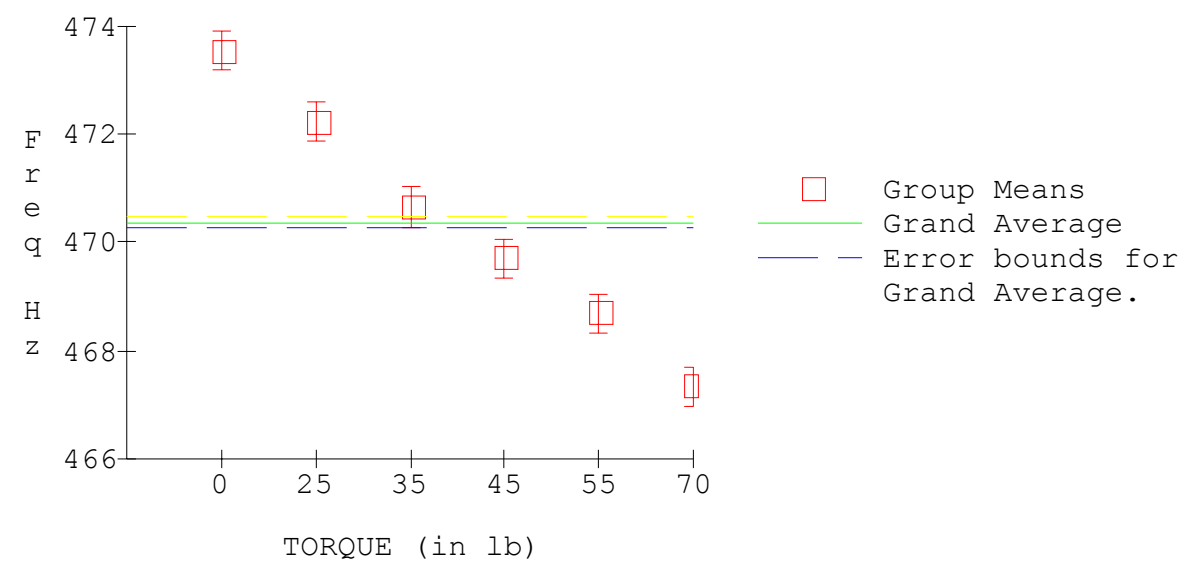

Figure 4. Holography-measured decrease in resonant frequency with increasing screw torque

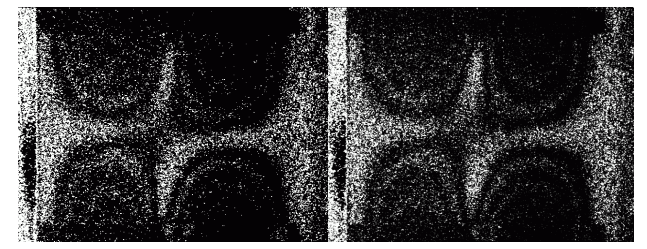

Figure 5. Mode at screw torques of 25 and 70 in. $1 \mathrm{~b}$ 
This example shows both the strengths and weaknesses of using wholefield interferometry for damage detection. Electronic time-average holography is easy to use to identify a vibration mode and its resonant frequency. ${ }^{10} \mathrm{~A}$ change-in-frequency is a commonly used signature of structural changes and damage. Resonant frequencies and modal identification are important design parameters for components such as fan blades. But the interferometervisualized mode shape of a component of a structure is not necessarily a reliable signature of structural health. A crack, de-bond, or other defect appearing in the region being visualized may indeed display a dramatic local effect on the fringe pattern or its derivatives as exemplified by figures 1 and 2 . Or it may not appear to change the pattern at all.

\subsection{Whole-Field Approaches}

Fortunately, electronic recording and computing enable another approach to damage detection from whole-field interference patterns. The entire pattern can be compared somehow with a pattern from the undamaged component $^{11-13}$ or even with a design-model-generated pattern. ${ }^{14-16}$ This approach does not depend on the presence of dramatic local structural effects such as cracks. A slight change in mode shape caused by a remote event, such as the change in screw torques associated with figures 3 through 5 , can be detected.

Artificial neural networks are one tool for parallel processing of wholefield interference patterns. A neural net can be trained to respond to very small changes in the total interference pattern. The simplest approach involves electronic time-average holograms. There is a technique for training neural networks to respond to very small changes in the shape of a vibration mode where the vibration mode is visualized using electronic holography. The technique assumes nothing about the nature or cause of the change in mode shape and uses modes of the undamaged structure for training.

Neural nets are used to automate the damage detection process and do not require visualization enhancements for human intervention. In that sense, they are consistent with the expectations for machine vision. The neural-net inspection technique is discussed in some detail next. 


\section{NEURAL-NET DETECTION OF DAMAGE}

\subsection{Neural-Net Essentials}

Artificial neural networks were rediscovered and started to assume some popularity about 15 to 20 years ago. ${ }^{17}$ It turns out that it is not especially straightforward to get good computing performance from neural networks. In fact it is not particularly easy to define an artificial neural network; since the number of different neural-net architectures is large. The principal requirements for getting good performance are to understand the intended application thoroughly and to select an appropriate architecture. Even then there is a confusing array of competing parallel processing methods with names such as fuzzy logic, expert systems and data mining. A single neuralnet architecture might have several training procedures.

Fortunately certain kinds of artificial neural networks handle the patterns generated from electronic holograms very well and can be trained to detect damage from these patterns. The so-called feed-forward artificial neural networks are especially well suited for training for damage detection from mode-shape patterns such as shown in figure 3 . The feed-forward neural net embodies most of the key concepts of an elementary neural network; can be trained by executing a formula procedure such as the back- propagation technique; ${ }^{18}$ and tolerates the laser speckle effect exhibited by electronic holograms of diffusely reflecting structures. In fact the feed-forward net handles noise very well and conceivably could be trained to distinguish damage from random, but normal, structure-to-structure variations. The feedforward net is not the only architecture for interpreting fringe patterns, but actually is quite compact in software when compared with other architectures. The feed-forward net is completely adequate for the damage-detection application and is discussed exclusively in the following paragraphs.

Figure 6 shows schematically a feed-forward net, sometimes called a multi-layer perceptron for historical reasons. The architecture consists of processing nodes or neurons represented by circles. The nodes are arranged in layers. Most often information is transmitted in one direction only from left to right; hence, the net is called a feed-forward net. The nodes in one layer are connected to the nodes in the previous layer via weighted connections. These weighted connections are sometimes identified loosely with biological synapses. Typically the weighted inputs are summed and then transformed with a non-linear transfer function. Sigmoid and hyperbolic tangent functions are typically used for the transformation. The transformed output is then transmitted to the next layer of nodes for similar processing. The single output of a node is sometimes loosely identified with a biological axon. 


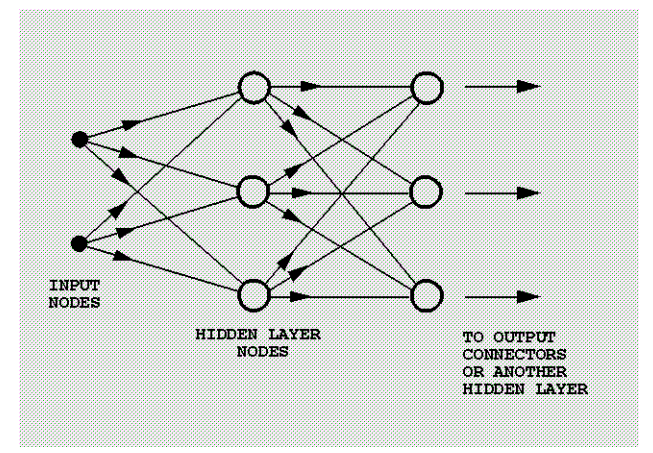

Figure 6. Example of feed-forward neural network

The nodes in the leftmost layer in figure 6 have single inputs to receive, for example, the pixel values from a fringe pattern. The outputs of the rightmost nodes are the outputs of the neural network. The layers in between are sometimes called hidden layers. A single hidden layer is most common. Occasionally a net will perform better with two hidden layers. The neural net can be trained to respond to specific inputs by outputting specific outputs. A set of records consisting of input-output pairs is used to train the neural network. Training is accomplished by adjusting the weights of the weighted connections to the nodes. One training criterion is that the sum of the squares of the differences between the actual neural-net outputs and the training outputs be minimized for all the training records. Training typically occurs in steps. A training step may involve all, a subset, or one of the training records. The best-known procedure is the so-called back-propagation algorithm. That procedure is essentially a steepest descent search in weight space to minimize the sum-of-squares mentioned above.

There is a vast literature on the details and vagaries of the various neuralnet architectures and training procedures. Ripley presents an exhaustive treatment of the mathematical subtleties. ${ }^{19}$ Unfortunately it is possible to read much of that literature without learning how to use nets to handle particular kinds of data. Fortunately there are free and commercial software packages for creating and training neural networks ${ }^{20}$ and ample opportunity to discover by experience which procedures work. The next section discusses the essentials for using the feed-forward net to recognize fringe patterns. 


\subsection{Design of Neural Nets and Training Sets for Damage Detection}

The rules for designing both neural nets and training sets for fringepattern recognition were evolved originally from computer experiments. The experiments were conducted using model-generated fringe patterns that included a model-generated speckle effect. ${ }^{14-16}$ The modeled object was initially a simple ideal cantilever, and the displacement distributions of the vibration modes were computed from a finite-element model. Modes were generated for cracked and undamaged versions of the cantilever, and multiple uncorrelated speckle patterns were calculated for each mode. The early experiments actually used as outputs the computed second derivatives of the model-generated dot product of displacement and the interferometric sensitivity vector. This choice of output specifically recognized that bending induced surface strain is proportional to the local second derivatives of displacement. ${ }^{8}$ The latest examples simply use a mode-identifying index called the degradable classification index (DCI) as the neural net output.

In any case, the current objective is that a neural net be trained to recognize a vibration mode of an undamaged structure in the presence of the laser speckle effect, and that the net respond to any changes in the mode shape. A property of neural networks called graceful degradation makes them useful for characterizing changes in the mode shape. Specifically, the proper design of the net and the training set will lead to a gradual and possibly controllable change in the DCI as the mode shape changes. Mode shape $^{21}$ depend on boundary conditions, distributions of material properties, excitation levels and damage.

The proper design techniques were deduced from the model-generated data using statistical analysis. Figure 7 shows some examples of measured and model-generated data from the first chord-wise mode or lyre mode of a flat blade. Figure 7(a) was photographed from the reconstruction from an old silver-halide time-average hologram of a vibrating blade. Figure $7(\mathrm{~b})$ was obtained from electronic holograms of a flat blade. Note the reduced spatial resolution caused by the relatively large camera pixels. Figure 7(c) was calculated from a finite element model of the flat blade and a model of the laser speckle effect. Note that the mode shape or characteristic pattern is displayed only for each of the 903 finite-element nodes and is portrayed as large pixels. Finally, figure 7(d) shows a measured pattern, sub-sampled from electronic holograms, at the same amplitude and with the same number of pixels used to generate figure $7(\mathrm{c})$. 


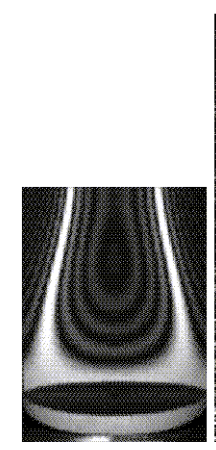

(a)

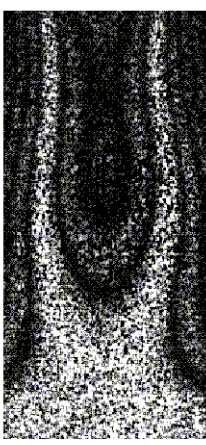

(b)

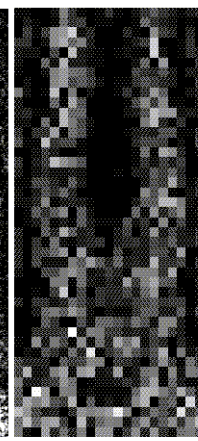

(c)

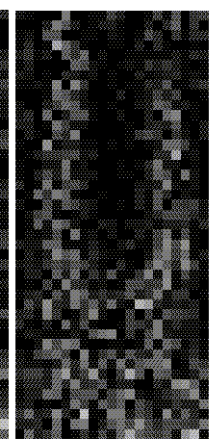

(d)

Figure 7. Measured and model generated chord-wise modes. (a), (b), and (d) are measured and (c) is model generated. Both (c) and (d) are sub-sampled.

The first thing to note is that the input data for the neural net is supplied at less than CCD-camera resolution and, in fact, at finite-element-model resolution. A few hundred to a few thousand finite elements are typically used for a compressor blade design. The second thing to note is that the patterns in figures 7(c) and 7(d) look quite similar. In fact, the performances of the neural networks have been quite comparable for model-generated and experimental data. The author is quite confident in changing from one kind of data to the other in testing neural-net behavior. But the fringe patterns predicted from finite-element models are not necessarily accurate themselves. Considerable work in the development of these finite-element models needs to be done, if neural nets are to be used to compare finiteelement structural designs with measured mode shapes.

Most often the patterns modeled or measured have been the characteristic patterns of classical time-average holography. The hologram is recorded, in principle, during at least one cycle and, in practice, over several cycles of vibration. Most simply subtracting two identical holograms whose reference beams differ only by a phase shift of $\pi$ generates the characteristic pattern. The mathematical form of the model-generated patterns, and ostensibly the measured patterns, is given by

$$
\text { (Speckle Pattern) } * J_{0}(2 \pi \vec{K} \bullet \vec{\delta})
$$

Here, $K$ is the sensitivity vector or the difference between the incidentlight-ray vector and the reflected-light-ray vector, all divided by the wavelength. The vibration displacement amplitude is denoted by $\delta$. The symbol $J$ denotes a Bessel function. The speckle pattern is generated from a 
model of the speckle effect by using the random number generators of the software package. The pattern represented by equation (1) can be signed or unsigned, but selecting the absolute value is convenient for visualization. For model-generated patterns, the displacement is computed at each node of the finite-element model.

A measured pattern is likely to be corrupted by a number of additional effects. These include electrical pattern noise, non-uniformities in illumination, pixel saturation and differences in the two holograms used to calculate the patterns. In practice, the net has proven to be reasonably immune to these effects. The feed-forward net is quite effective at handling Gaussian noise sources. The patterns can be averaged to improve the signalto-noise ratio for speckle-effect and electrical noise, but the objective has been to use two holograms per pattern when possible. Processing is done with software, and the combination of 2-hologram patterns and finiteelement resolution has permitted 30 -frame-per-second visualization.

The rules to be stated for the net and training-set designs also apply to double-exposure electronic holograms. An additional complication in double-exposure holography is synchronization or the point in the vibration cycle where the two exposures are recorded. Fluctuations constitute an additional noise source. The remainder of this chapter discusses timeaverage holography exclusively.

The design rules are based on results of statistical design-of-experiments and response-surface methods ${ }^{22}$ as well as experience. In effect, the neural network is treated as a black box, and its response is measured experimentally. Among the factors that could be considered are: the number of independent or uncorrelated speckle patterns per mode, the number of distinct vibration modes or classes of modes, the number of input nodes, the intensity range of the test patterns, the accompanying pixel saturation effects, the vibration amplitude and number of characteristic fringes, the transfer functions of the neurons, additive electrical noise, the nature of the output code, the number of hidden layers, the number of hidden layer nodes, and the coding, conditioning and transformation of the input patterns.

Early experiments measured performance as a function of the number of speckle patterns per mode, the number of hidden layer nodes, pixel saturation, and the size of a modeled crack. ${ }^{14}$ A later study showed that the coding, conditioning and transformations of the input patterns affected significantly the magnitude of the minimum detectable change in the vibration amplitude distribution. ${ }^{13}$ The results of these studies have proven to be effective for both model and experimental data. The design rules listed next were developed for feed-forward nets that contain one hidden layer. ${ }^{14}$ 
First, the neural nets are called sparse, in that they contain few hidden layer nodes. In general, 3 hidden-layer nodes are used for each distinct mode or class of modes to be learned. For example, a net can be trained to learn a particular mode for both cracked and uncracked versions of a cantilever by using a net with 6 hidden-layer nodes. The vibration level at the tip is the same for both the cracked and uncracked versions. Or a net can learn to assign one mode to a single class and several other modes to another class, if that net has 6 hidden-layer nodes ( 3 for each class). The class codes, in this case, are called degradable classification indices (DCI). The reason for this terminology is that a change in the mode shape of the mode in a class by itself decreases the corresponding node output. Typically each class has its own output node. This property is used to implement the whole-field damage-detection technique. The rule for the number of hidden-layer nodes is not exact. In some cases, fewer than 3 hidden-layer nodes per class are effective. The use of more hidden-layer nodes has little effect. A sparse net can have large numbers of input and output nodes without taxing the software; hence, sparse nets are convenient for processing images and fringe patterns with large numbers of pixels. Real-time (30-frame-per-second) processing can be maintained.

Second, there must be enough uncorrelated speckle patterns per mode for the net to learn to ignore the speckle effect. In effect, the net has to see enough independent noise samples to learn to treat speckle-effect noise as irrelevant. An effective rule is to select uncorrelated speckle patterns equal in number to 10 percent of the number of input pixels. A typical pattern, for example, would contain 2000 sub-samples. A training set would then contain 200 uncorrelated speckle patterns per mode.

Third, the net can tolerate some saturated inputs. Unsigned inputs typically occupy the range 0 to 1 for the feed-forward nets. By contrast, the hidden-layer and output-layer nodes typically use the S-shaped sigmoid transfer function. The sigmoid function approximates the unit step function. Output codes are normally chosen to be $[0.2,0.8]$ instead of $[0,1]$; since the latter values would require sigmoid-transfer-function arguments of minus and plus infinity.

The tolerance of input-pattern saturation was proven by multiplying the inputs by constant saturation factors and clipping the inputs greater than 1 . The results were not affected even when the saturation parameter was as large as 4 . No doubt, the large number of unclipped darker speckles was the reason that such a large saturation factor could be tolerated. 
Fourth, the preparation and conditioning of input patterns can have a significant effect on the ability of the net to learn to distinguish patterns. ${ }^{13}$ Early experiments with the raw absolute value patterns represented by equation (1) had difficulty distinguishing the model-generated cracked and uncracked cantilever modes. It was necessary to amplify the difference in displacement distributions, before the net would learn the difference. A data transformation technique called folding ${ }^{13}$ changed that result significantly. Following folding, the crack could be de-amplified, and the net would learn to detect it.

Folding is an intensity dependent transformation of the raw input data. Consider the signed characteristic pattern, generated from one model of the speckle effect, given by:

$$
\text { mode }=\mathrm{A} \cos (\Theta) \mathrm{J}_{0}(2 \pi \overrightarrow{\mathrm{K}} \bullet \vec{\delta})
$$

In equation (2), $\mathrm{A}$ is a positive random quantity, and $\Theta$ is a random number uniformly distributed between 0 and $2 \pi$. The sub-sampled signed quantity can be used to train the net, or the data can be folded before training. The folding transformation is shown in figures 8 and 9 for one and 3 folds, respectively. Signed input data are normalized in the range $[-1,1]$ and unsigned input data are normalized in the range $[0,1]$. One fold is equivalent to taking the absolute value of the pattern in equation (2). The contrast of the transformation can be reversed as well.

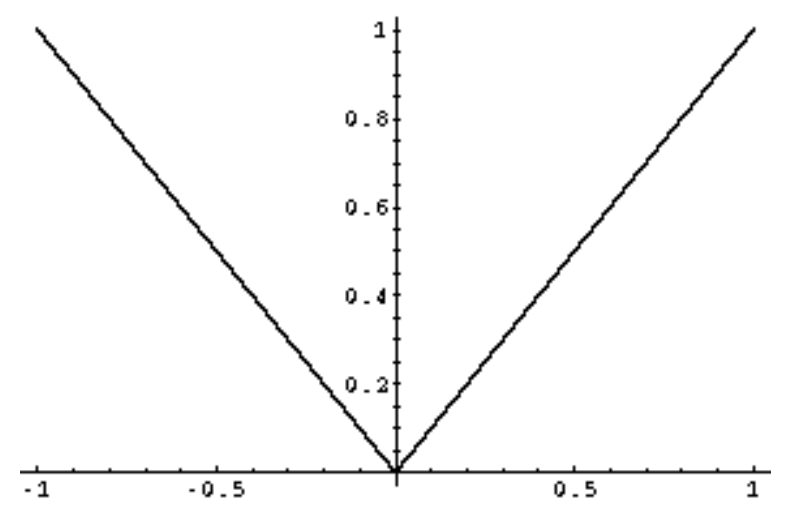

Figure 8. Intensity data folded once. Equivalent to absolute value. 


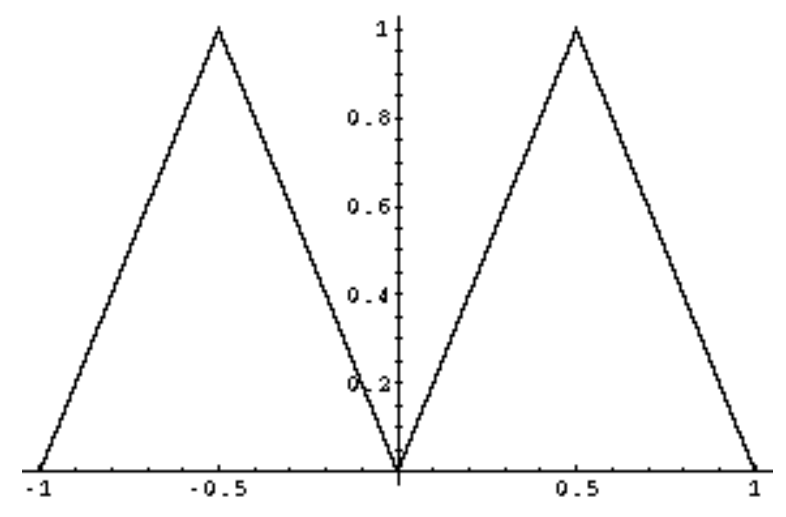

Figure 9. Intensity data folded three times.

The effectiveness of folding is proven for the blade and finite-element node- pattern shown in figure 10. Three copies of the blade shown in the upper right of figure were manufactured. One of the blades had a small crack in its base. Finite-element models were created for both the cracked and undamaged blades. A neural net was trained using the mode shapes computed from the finite-element models, using various numbers of folds. The training errors are shown to decrease as the number of folds increases up to 8 . Folding increases the sensitivity of the neural-net inspection process for identifying cracks. The crack amplitude difference was actually deamplified by a factor of 10 for this demonstration. A training error, a test error (obtained from different speckle patterns than used for training) and a test percent-error-rate are shown as a function of the number of folds.

Other kinds of data transformations and preparation have been used to improve learning. Stretching individual pixel intensity distributions into the same intensity range is sometimes used, but leads to over fitting and the tendency to learn the noise rather than to learn to ignore it. A net that uses more general transformations of input data is the Functional Link Net. ${ }^{18}$ But folding handles the damage detection application very effectively and in a most simple manner. Nets have been trained using folding to detect changes in displacement distributions, where the maximum displacement change was as little as 10 nanometers.

The suggested net and training-set design rules can be verified using model-generated data. The next section discusses briefly how to record experimental speckle patterns for training. Experimental training records are required for the damage detection method to be discussed shortly. 

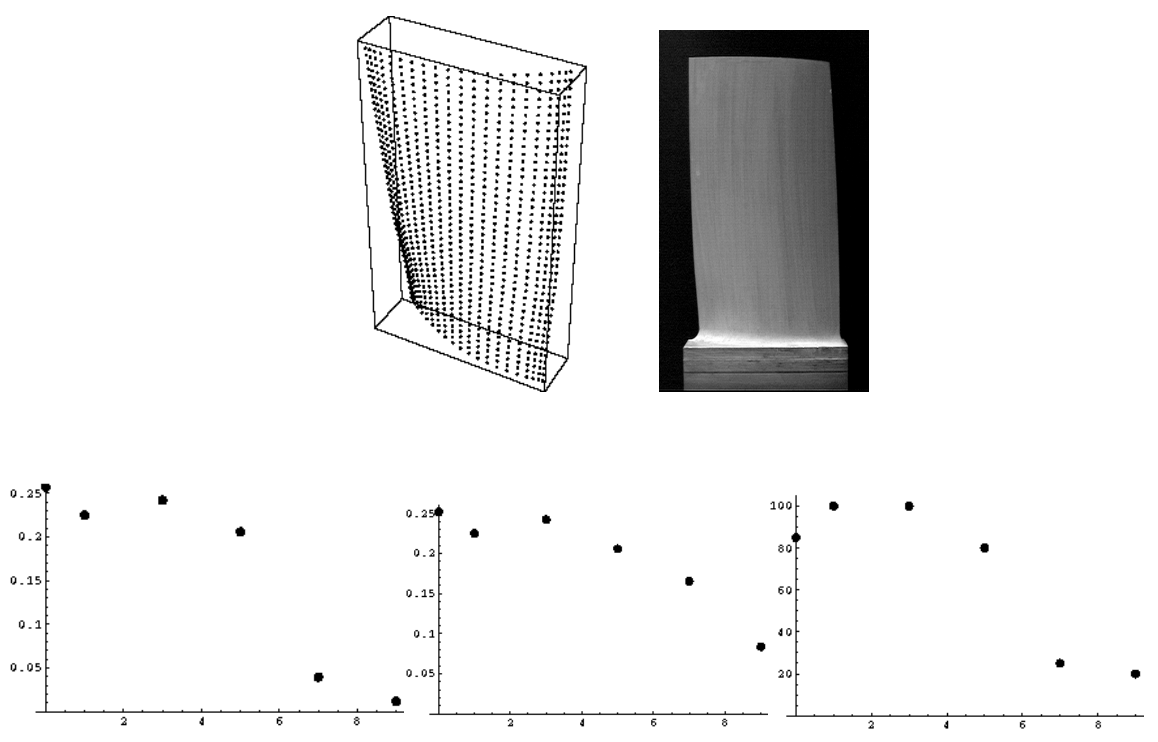

Figure 10. Training error, test error and error rate versus number of folds for finite-element-model data generated for the blade and node pattern shown

\subsection{Experimental Training Sets}

The recording of experimental patterns for training a neural net to detect structural damage and changes, or to compare with computationally generated patterns, is especially advantageous. Experimental recording of sub-sampled patterns automatically encompasses the vagaries of the holography rig, including beam fluctuations and non-uniformities, electrical noise, illumination and reflection variations, difficult-to-model artifacts, random material and geometrical effects, ill-defined boundary conditions, and any of the hidden effects that are easily overlooked in modeling.

The major requirement is to record enough uncorrelated speckle patterns according to the second rule in section 3.2. Physically, speckle patterns do not change rapidly enough, without external inducement, to accomplish this efficiently, although they do vary with vibration-induced beam changes and the like. Fortunately, the large pixels solve that problem. Measurements are made at random positions within the large pixels, such as make up figure 7(c), to build up the sub-sampled input array. A single full-resolution characteristic pattern could, in principle, be used to generate an entire training set of sub-sampled patterns. But selecting a new characteristic pattern each time a training record is assembled allows the other vagaries to manifest themselves. In any case, nets trained in this manner tolerate variations in the speckle effect as well as do model-trained nets. 
It should be noted that data acquired to compare with finite-element models will need to be consistent with the positional variations of the element size. Figure 10 shows how the spacing of the nodes in a finiteelement pattern can vary.

The next section discusses how to use the material of sections 3.2 and 3.3 to train a neural net to detect structural damage or structural changes.

\subsection{Training Neural Nets to Detect Structural Damage by Using the Mode Shapes of the Undamaged Structure}

The following procedure is designed to accompany a possibly destructive test of a structure or a test where structural changes might occur. Examples include, but certainly are not limited to, pressure-cycle-induced damage, fatigue failure, de-bonding in composite structures, loose fasteners, and bearing changes. The assumption is that the structure is available for holographic vibration-mode analysis before conducting the possibly destructive test. The procedure uses only the vibration modes of the unchanged or undamaged structure for training. ${ }^{11,12}$

First, use electronic holography to identify about 5 vibration modes of the structure to be inspected.

Second, establish that parameters such as fastener torques, object alignment, excitation, and amplitude measurement are under control. The net will detect effects of boundary conditions, for example, resulting from changes in fastener torques. The holographic inspection is most easily done on a vibration-isolation table. It may be convenient to conduct both the inspection and the possibly destructive test on the same table. Otherwise, attention must be paid to realigning the object accurately relative to the holocamera for each inspection. Random alignment errors are a noise source, and feed-forward nets can learn to tolerate them to some extent. Field inspections are possible, and inspections have been performed using fiberbundle holocameras for remote access to an object. But it is still best and easiest to use the optical table.

Third, establish the sampling grid for the large pixels. Non-rectangular and curved objects may require large pixels that vary in size. For the sample applications discussed in section 4, the pixel coordinates of the edges of the object were measured through the holocamera and supplied as inputs to a grid-calculating software package. Typically 1,000 to 2,000 nodes and the associated large pixels are used. 
Fourth, select three vibration modes, and choose one of these modes as the mode to be monitored for structural changes and damage. This process might require some trial and error. The mode mix will determine the sensitivity of the process to some extent, and affords some choice, for example, in calibrating or quantifying the inspection.

Fifth, excite the vibration mode to be monitored for damage. The excitation level must be controlled. Fluctuations in the amplitude constitute a noise source. It is convenient to monitor a point on the object using a laser interferometer to assure that the vibration amplitude is controlled. An expert operator may also be able to set the amplitude adequately by viewing the characteristic pattern. Typically, the maximum excitation amplitude will not exceed one wavelength of light.

Sixth, record the required number of speckle patterns as discussed in section 3.2, and assign an output code for the mode to be monitored. For example, two output nodes might be employed. The output code might be $[0.8,0.2]$ for feed-forward nets as already discussed. The first number is the DCI of the pattern to be monitored. The other vagaries in the set-up and procedure automatically will be incorporated in setting the code.

Seventh, record the required number of speckle patterns as discussed in section 3.2 for the other two modes and for the zero-amplitude condition. Use another code for these patterns that places them in the same class. The output code might be $[0.2,0.8]$. In effect, these patterns are examples of patterns that differ from the pattern-to-be-monitored. Changes in the mode shape of the pattern being monitored will then result in a decrease in the DCI generated by the trained net.

Eighth, construct a feed-forward net with 6 hidden-layer nodes as discussed in section 3.2, and train the net to an RMS error of 0.01 or less. The 6 hidden layers support 2 classes. The RMS error is computed from the squares of the differences between the training outputs and measured outputs for all training records.

Ninth, test the net for sensitivity. Ideally, the net is calibrated or at least quantified to be consistent with certain structural test criteria. One approach is to make the neural-net test consistent with NASA vibration handbook standards. ${ }^{23}$ In the absences of calibration or quantification, simple judgment may be the only alternative. For example, light point loads can be applied at critical locations to test the neural net response, or fastener torques can be varied.

Tenth, change the vibration-mode mix, if the sensitivity is not correct, and repeat the above steps. The vibration-mode-to-be-monitored is the most critical. 
Eleventh, establish criteria for interpreting the results of the test. For example, a change in the DCI from 0.8 to 0.7 might be declared to be an indicator of damage. At 0.7 , an alarm can be triggered. One approach is to color the fringe pattern differently, when the DCI decreases below 0.7. A change of the fringe color from green to yellow or red can be used as an indicator. This is decidedly an ANOVA viewpoint. For noisy data, a regression of the DCI against some test parameter such as time or the number of vibration cycles or the number of pressure cycles might be more appropriate. A trend can be established and its significance measured. Interpretation will be discussed in more detail together with the applications. Experience has shown that these steps can be executed very quickly. The time required to record a training set, train the net, and link the net with other software was typically twenty minutes for the two sample applications to be discussed.

The first application of neural-net detection of damage demonstrates some of the statistical considerations involved in interpreting results and applying quantification or calibration techniques. The second application discusses an early use of unfolded neural-net technology to detect pressure-cycle-induced damage in an International Space Station instrumentation cold plate.

\section{SAMPLE APPLICATIONS}

\subsection{Optical Strain Gauge Mounting Plate}

This application demonstrates the ability of the neural networks to detect changes in a vibration mode shape in a region remote from the cause. In that sense, the application is a health monitoring application. The object is a plate used to mount and test optical strain gauges, ${ }^{9}$ and was discussed in section 2.2 in connection with changes in frequencies of vibration-modes. The frequency changes, in turn, were caused by a change in the torque of a mounting screw. Figure 3 showed a vibration mode of the plate mounted between two vise-grip mounts. Figure 11 shows the plate in one of the electronic holography arrangements used to measure it. As already stated, frequency changes were induced by adjusting the torque of the second screw from the top on the left.

The plate itself was rather thin at $813 \mu \mathrm{m}$, and was $152.4 \mathrm{~mm}$ wide and $101.6 \mathrm{~mm}$ high. Optical strain gauges typically are to be mounted along the center line, when the plate is to be used for that purpose. 
The key point is that only the plate itself, and not the vise-grips and screws, were visible to the neural nets. Hence, only the mode-shape change on the plate itself was relevant. Figure 12 shows the mode shapes as well as part of the mounting hardware. Screw torques were initially set to $70 \mathrm{in}$. $\mathrm{lb}$. The torque of the second screw from the top on the left was then backed off fully and set to $25 \mathrm{in}$. $\mathrm{lb}$ for training. The training sets, subsequently recorded, remained valid for weeks, provided that the torques were periodically re-adjusted to the initial values.

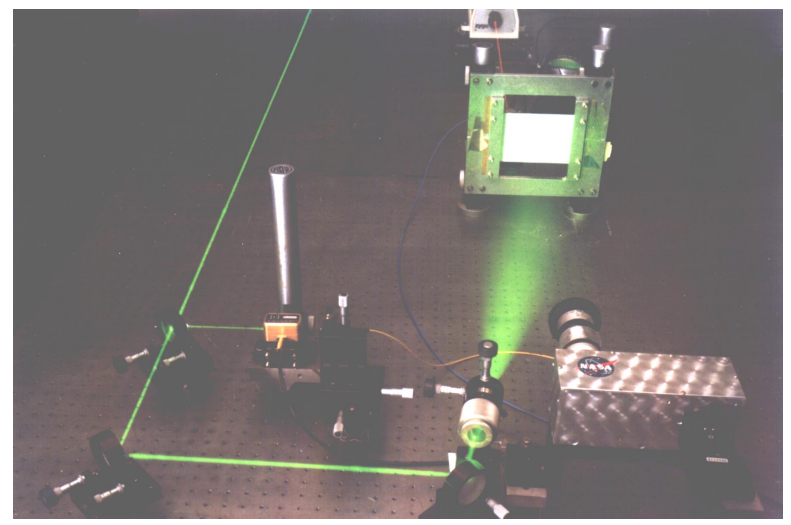

Figure 11. Optical strain-gauge plate in an electronic holography arrangement

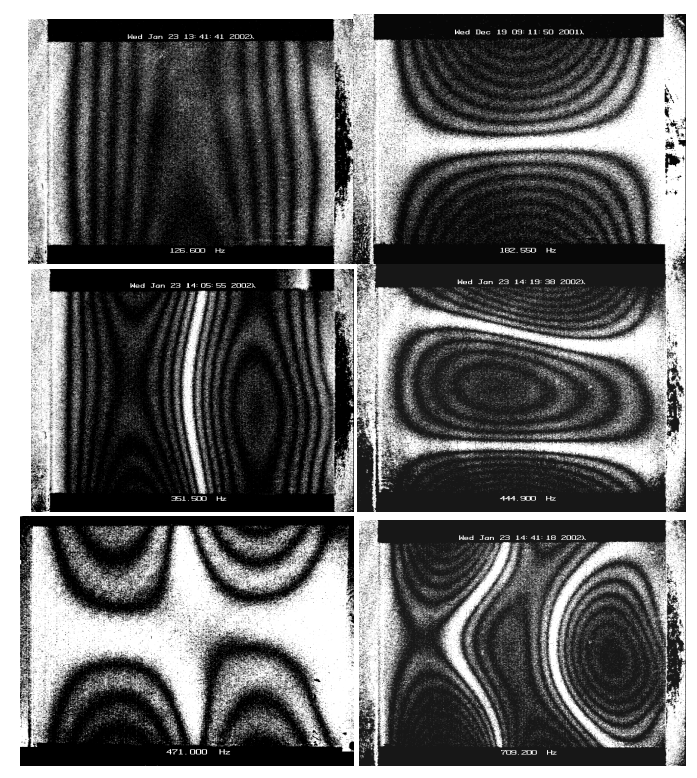

Figure 12. Mode shapes for optical-strain-gauge mounting plate. Resonant frequency increases left to right and top to bottom. 
The sampling grid was almost uniform, and was 42 nodes wide and 28 nodes high for a total of 1176 nodes. Hence, the neural net had 1176 input nodes. The full resolution of the image that was used for the inspections was 537 pixels wide and 395 pixels high. The software automatically adjusted the large pixels at the end of a row and the bottom to handle round off. The average large pixel then contained about 180 speckles from which the random patterns were generated.

The fifth mode at about $473 \mathrm{~Hz}$ was placed in a class by itself for monitoring; since the frequency changes of that mode were found to vary most strongly with changes in the mounting screw torque. The first mode, the second mode and the zero-amplitude condition were all selected as members of the second or comparison class. Each comparison mode was assigned the same output code.

A single point of the plate was monitored with a laser interferometer, so that the vibration amplitude at that point could be set and maintained at the same value. The point was located on the rear of the plate, $19 \mathrm{~mm}$ from the bottom and $29 \mathrm{~mm}$ from the edge of the left vise-grip as viewed from the holocamera side. The peak-to-peak amplitude was set and maintained at $0.32 \mu \mathrm{m}$ for the fifth mode at the laser-interferometer detection point. The fluctuation in the amplitude, due to various noise sources, was about $0.025 \mu \mathrm{m}$. That fluctuation consisted primarily of a superimposed first mode, and constitutes one of the vagaries of the training procedure. It can lead to a rather substantial variation in the monitored DCI; since the first mode was already assigned to the comparison class. Perhaps the first mode should be avoided as a member of the training set; since it is so easily excited. But the first mode was in fact included in the training set for this sample application.

Ten percent of the number of large pixels or input nodes is equal to 118 . Hence, in accordance with section 3.2, 118 sampled uncorrelated speckle patterns were recorded for the first, second, and fifth modes and the zeroamplitude condition. Needless to say the zero-amplitude condition contained a small amount of environmentally excited first mode, as did the other modes. The software automatically performed random selection of the speckles from one pattern to the next. The fifth mode was assigned the output $[0.8,0.2]$, and the other modes together were assigned the output $[0.2,0.8]$. For those cases where folding was employed, the input data were also subjected to a 5 -fold folding transformation as discussed in connection with figures 8 and 9.

A feed-forward net was then constructed in software. It consisted of 1176 input nodes, 6 hidden-layer nodes with sigmoid transfer functions, and 2 output nodes with sigmoid transfer functions. The net was then trained to an RMS error less than 0.01 . 
The trained net was converted into C-language code and compiled and linked to the software of the electronic-holography inspection system. As mentioned before, the entire process requires about 20 minutes.

\subsection{Statistical Results}

Tests variously involved recording 10 uncorrelated speckle patterns per torque setting and recording 100 uncorrelated speckle patterns per torque setting, each averaged 10 times for electrical noise.

The net had no trouble detecting changes, even in a $10 \mathrm{in.}$ lb interval, when the amplitude was not reset to its constant value of $0.32 \mu \mathrm{m}$. But the mode shape does not change much, as stated before, at a constant amplitude setting. Nevertheless, the neural nets did detect these mode-shape changes. Figure 13 shows the results of an ANOVA performed on 100 uncorrelated speckle patterns per torque setting. Each of these patterns was averaged 10 times for electrical noise, random first-mode vibration noise and other noise sources. The DCI can be observed to decline as the screw torque is increased from the training value of 25 to $70 \mathrm{in}$. $\mathrm{lb}$. The Bonferroni simultaneous confidence intervals are clearly separated. The most important point is that this test was repeated several times over a 2-week period using the same training set. The DCI decreased significantly in all cases. Hence optical alignment effects, and other systematic and random effects, were not critical. An F Value test of significance indicated that the probability of the observed variation being due to random chance was less the 0.001 .

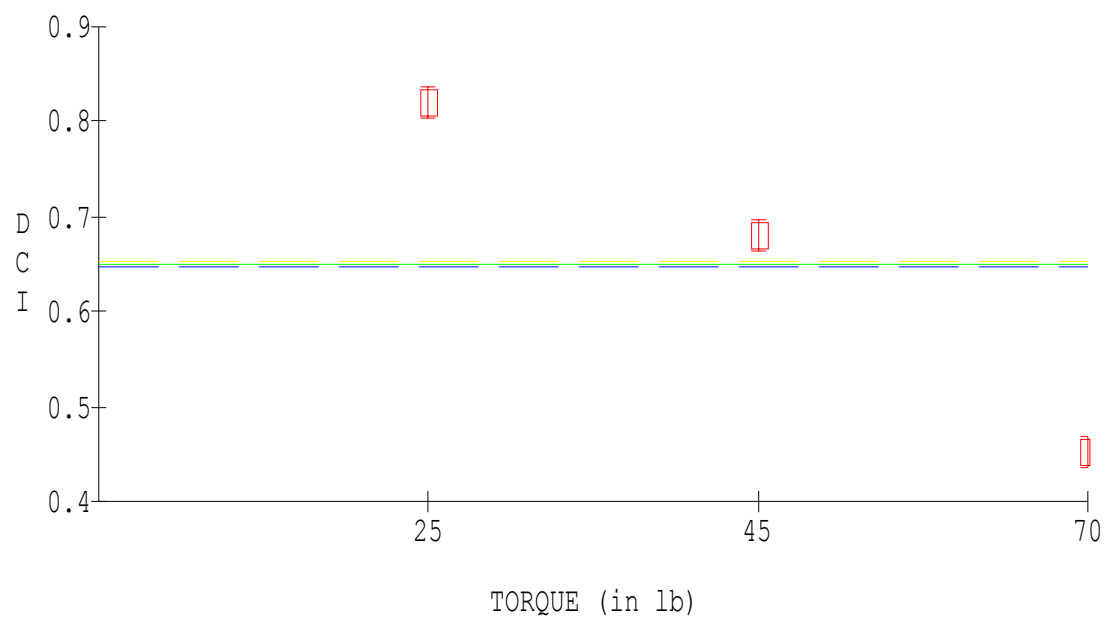

Figure 13. Neural-net response to change in mode shape induced by a change in screw torque. 
The above data were also tested with a linear regression using the screw torque as the only factor. This test provides a better measure of the scatter of the data and goodness of fit. The $\mathrm{R}^{2}$ value was 0.75 indicating quite a bit of scatter. But the T-value for the slope was almost -30 indicating that the significance of the slope was much better than 0.001 . The slope is given by -0.008202 (in. $\mathrm{lb})^{-1}$.

Regression also allows the lower detection limit to be quantified. For that purpose, a trend was sought over a $10 \mathrm{in}$. $\mathrm{lb}$ range only from 25 to $35 \mathrm{in}$. $\mathrm{lb}$. Ten patterns were recorded at each station without averaging, and there were stations at 25, 31, 34, and $35 \mathrm{in}$. lb. The input data were folded 5 times in the manner of figures 8 and 9 . The slope obtained from these measurements was -0.008636 (in. $\mathrm{lb})^{-1}$, but $\mathrm{R}^{2}$ was only 0.085 , and the significance of 0.069 was poorer than the old industrial standard for significance in statistical testing (0.05).

For this application, crisp red-lining of changes in the mode shape induced by changes in the screw torque required more than 10 patterns per station with station separations of $10 \mathrm{in.} \mathrm{lb}$ or more. Crisp results required 100 patterns per torque value. Trends could be established with less data by using regression. But both regression and ANOVA require repeat measurements because of noise. Hence, visualization needs to proceed much more slowly than 30 frames per second. An application that was accomplished at the full visualization rate with unfolded training data is discussed next.

\subsection{Inspection of International Space Station Cold Plate}

This application ${ }^{11}$ was performed before the discovery of the folding technique; hence a more sensitive test might have been possible. Nevertheless, this application defines the kind of test that is intended for damage detection from neural-net inspection of fringe patterns. The application was conducted with real-time visualization as well as by examining repeat samples. The subject was an International Space Station (ISS) instrumentation cold plate shown in figure 14. The cold plate had holes for mounting instrumentation and internal cooling passages for cooling the instrumentation. It was suspected that the cooling passages might not withstand pressurization; hence it was decided to perform pressure cycling of the cold plate and inspect the cold plate for ensuing damage. The cold plate was in fact inspected with a variety of techniques including classical silver-halide-emulsion holography and ultrasonic C-scan imaging. Only the neural-net inspection is discussed. A key point is that the holographic nondestructive inspection and the potentially destructive test were performed 
simultaneously on the same vibration isolation table. Another point is that the decision of the net was crisply reported. That is, the DCI was noted only as being greater than or equal to a redline condition. The monitored fringe pattern was colored green for a DCI greater than or equal to 0.65 and yellow otherwise.

The cold plate was bolted to a frame through several of the holes intended for mounting instrumentation. The cold plate was filled with water and connected to a pressure cycling source. Neural net inspections were performed before, during and after pressure cycling. Both the entire plate and a suspicious region between four boltholes were inspected. The method of 3.4 is discussed only for the inspection between the four boltholes. The boltholes are on 4 in centers.

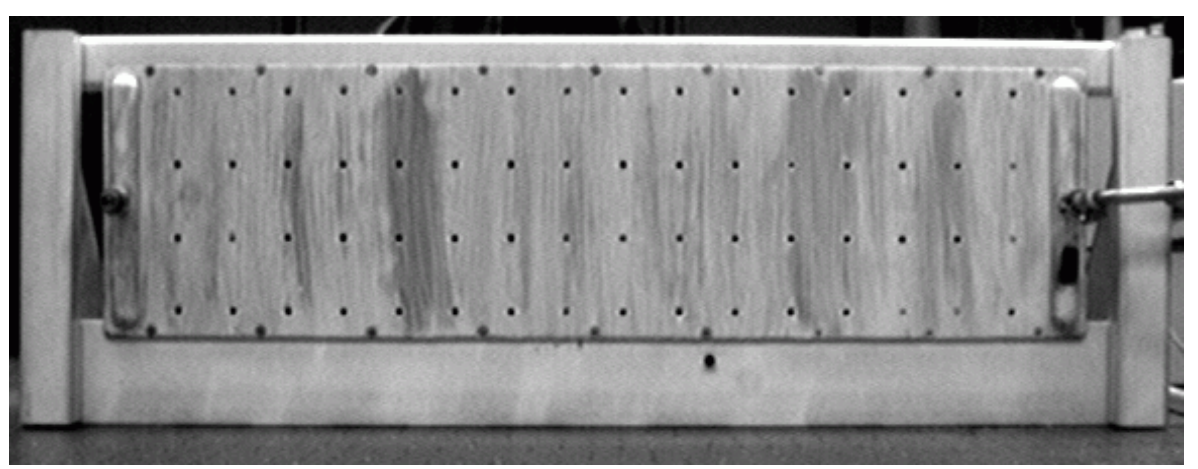

Figure 14. ISS cold plate from front

Figure 15 shows five modes covering a region including the suspicious region between the four boltholes.

Fasteners were set, and the cooling passages were connected to the pressure source at $120 \mathrm{psig}$. The source could be cycled at $0.2 \mathrm{~Hz}$.

The sampling grid consisted of 40 by 54 large pixels. Figure 16 shows the large-pixel pattern at zero amplitude.

The selection of an adequate set of training modes actually required more than one attempt. The selection process involved applying slight point loads to the cold plate and observing the neural-net response. Clearly a statistical approach or a calibration would have been preferable. The mode at $2310 \mathrm{~Hz}$ was found to be most sensitive to perturbations, and that mode was selected for inspection. The modes at zero amplitude, 780 and $1597 \mathrm{~Hz}$ were used for the comparison class. A laser interferometer controlled the vibration amplitude. 


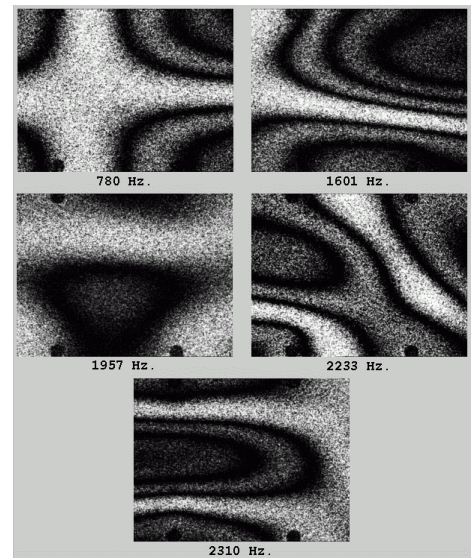

Figure 15. Modes covering region between four bolt holes

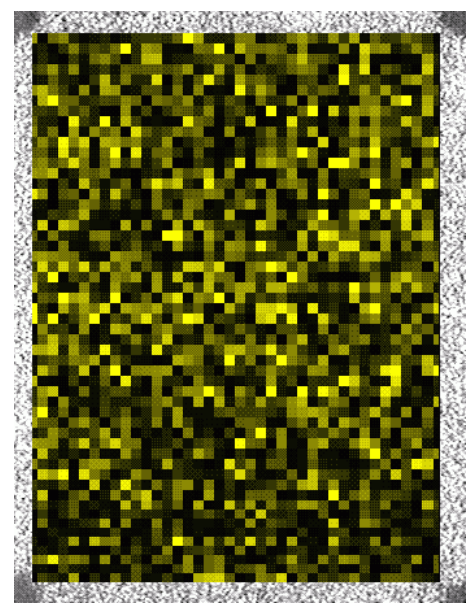

Figure 16. Large-pixel sampling pattern between four bolt holes

There were 2160 large pixels covering a region of 314 by 423 small pixels. Hence the training set for each mode contained 216 uncorrelated speckle patterns.

The feed-forward net contained one hidden layer and that layer contained 6 hidden-layer nodes as discussed before. Training proceeded quickly. As stated the process of section 3.4 needed repeating before the most sensitive training set was found.

The trained net was then used during a 1000 pressure-cycle test and inspection of the region between the boltholes. A 2000 pressure-cycle test had been conducted previously for the whole plate. The plate was continuously monitored during the test, even during pressure cycling. 
Pressure cycling was interrupted every 250 cycles to record 20 neural-netprocessed characteristic patterns at the pressure-relaxed condition.

The net flagged a significant change in mode shape at maximum pressure, but always flagged an insignificant change at the pressure-relaxed condition. Figure 17 shows the mode between the bolts for the pressurerelaxed and pressurized conditions. Note that only part of the mode shown in figure 15 actually appears between the boltholes.

The conclusion of this test was that the neural-net inspection of mode shapes did not show significant changes or damage from pressure cycling. This conclusion was in agreement with the conclusions reached using ultrasonic C-scan testing.

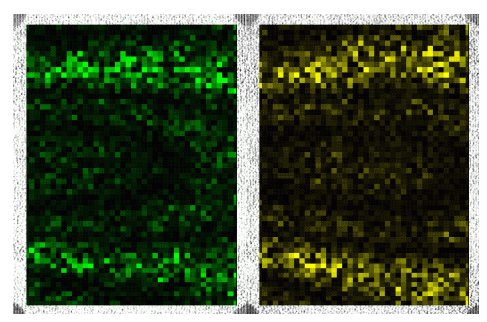

Figure 17. Response of neural-net to pressure-relaxed and pressurized conditions.

A DCI less than 0.65 is coded yellow in displaying the fringe pattern.

\section{SUMMARY AND FUTURE TRENDS}

Whole-field laser holography and interferometry are non-destructiveevaluation (NDE) methods. They are used to determine whether a component, structure, or system changes from its design condition during normal operations or other testing. The NDE method is non-invasive and does not itself change the structure.

Electronic recording of interference patterns makes the inspections much more efficient. The data are also available immediately for computer analysis.

Neural-net processing allows the entire fringe pattern to be examined simultaneously and in parallel. The inspection process and ensuing decisions are automated. The approach reported in this chapter is essentially a formula procedure. It can be consistent from one application to another and from one laboratory to another. It is subject to calibration and quantification. It provides a solid answer to a question that has been asked since laser holography was discovered: "Now that we have all this data, how do we interpret and use it?" 
The ultimate goal, and one of the original goals for the neural-net processing of fringe patterns, was to create an efficient interface between damage models and data. A computational model of a structure and an optical model of an inspection process can be used to generate training records. The trained net can be then be used to examine experimental patterns. The inverse process of using experimentally trained nets to examine model-generated patterns also is relevant. So far the structural models used have not been accurate enough, in general. But the noise immunity of the feed-forward net and the availability of probabilistic finiteelement models ${ }^{24}$ offer future hope for the model interface.

The technology of damage detection using neural-net inspection of fringe patterns is definitely good enough to be placed in the hands of designers and NDE specialists. The technology constitutes a standard that their models and procedures hopefully will meet, eventually. The most straightforward approach is to incorporate the neural-net procedure into existing standards for vibration testing. ${ }^{23}$ The response of the net and the responses of standard sensors such as strain gauges and displacement sensors can be correlated.

Most of this chapter has discussed the fringe patterns from electronic time-average holography. But the general procedure of section 3.4 certainly can be adapted to other kinds of damage sensitive patterns. These patterns can be obtained variously from speckle photography and interferometry, moiré deflectometry, thermography, and temperature sensitive paints, large angle scattering, and any other image forming method that is affected by damage. The algorithmic approach of section 3.4 could be applied virtually unchanged.

The technological limit of the neural-net inspection technique is that it based in software and is limited to a few thousand sub-samples. An improvement would require efficient channels for direct neural-net processing of optical data. 


\section{REFERENCES}

1. Y. Bar-Cohen, ed., Automation, Miniature Robotics and Sensors for Nondestructive Evaluation and Testing, Vol. 4, Topics On Nondestructive Evaluation, B. B. Djordjevic and H. D. Reis , The American Society for Nondestructive Testing, Inc., Columbus, OH (2000).

2. C.M. Vest, Holographic Interferometry, Wiley, New York (1979).

3. R.J. Collier, C.B. Burckhardt and L.H. Lin, Optical Holography, Academic Press, New York (1971).

4. Ref. 1, 125-126.

5. S.A. Benton, ed., Practical Holography VI, Proc. SPIE 1167 (1992).

6. Ref. 3, 451.

7. A.J. Decker, "Holographic interferometry with an injection seeded Nd:YAG laser and two reference beams," Appl. Opt. 29(18), 2696-2700 (1990).

8. Ref. 2, 147-177.

9. J. Lekki, G. Adamovsky, P. Flanagan and K. Weiland, "Evaluation of mechanical modal characteristics using optical techniques," NASA TM 2002-211678 (2002).

10. K.A. Stetson and W.R. Brohinsky, "Electro-Optic Holography System for Vibration Analysis and Nondestructive Testing," Optical Engineering 26(12), 1234-1239 (1987).

11. A.J. Decker, M.E. Melis and K.E. Weiland, "Inspection of Space Station Cold Plate Using Visual and Automated Holographic Techniques," NASA TM 1999-209388 (1999).

12. A.J. Decker, "Neural-Net Processed Characteristic Patterns for Measurement of Structural Integrity of Pressure Cycled Components," Proceedings of JANNAF, $12^{\text {th }}$ Nondestructive Evaluation Subcommittee, Cocoa Beach, Florida (2001).

13. A.J. Decker, "Optimization of training sets for neural-net processing of characteristic patterns from vibrating solids," Proc. SPIE 4448, 209-217 (2001).

14. A.J. Decker, E.B. Fite, O. Mehmed and S.A. Thorp, "Processing speckle patterns with model trained neural networks," Proc. SPIE 3172, 285-293 (1997).

15. A.J. Decker, E.B. Fite, S.A. Thorp and O. Mehmed, "Comparison of computationalmodel and experimental-example trained neural networks for processing speckled fringe patterns," International Conference on Optical Technology and Image Processing Fluid, Thermal, and Combustion Flow, Proc. VSJ-SPIE '98, on compact disc in pdf, paper AB002 (1998).

16. Ref $1,309-321$.

17. D.E. Rumelhart, J.L. McClelland, and the PDP Research Group, PARALLEL DISTRIBUTED PROCESSING, Explorations in the Microstructure of Cognition, Volume 1: Foundations, MIT Press, New York (1986).

18. Y-H. Pao, Adaptive Pattern Recognition and Neural Networks, Addison-Wesley, New York, 120-129 (1989).

19. B.D. Ripley, Pattern Recognition and Neural Networks, Cambridge University Press, Cambridge (1996).

20. Examples in this chapter were created using NeuralWorks Professional II Plus.

21. W.C. Hurty and M.F. Rubinstein, Dynamics of Structures, Prentice-Hall (1964).

22. Statistical analysis in this chapter was performed with $R S /$ Suite software.

23. "Dynamic Environmental Criteria," NASA-HDBK-7005 (2000).

24. W.K. Liu, T. Belytschko, and Y.J. Lua, "Probabilistic Finite Element Method," Probabilistic Structural Mechanics Handbook, Theory and Industrial Applications, C. (Raj) Sundararajan, ed., Chapman \& Hall, 70-105 (1995). 


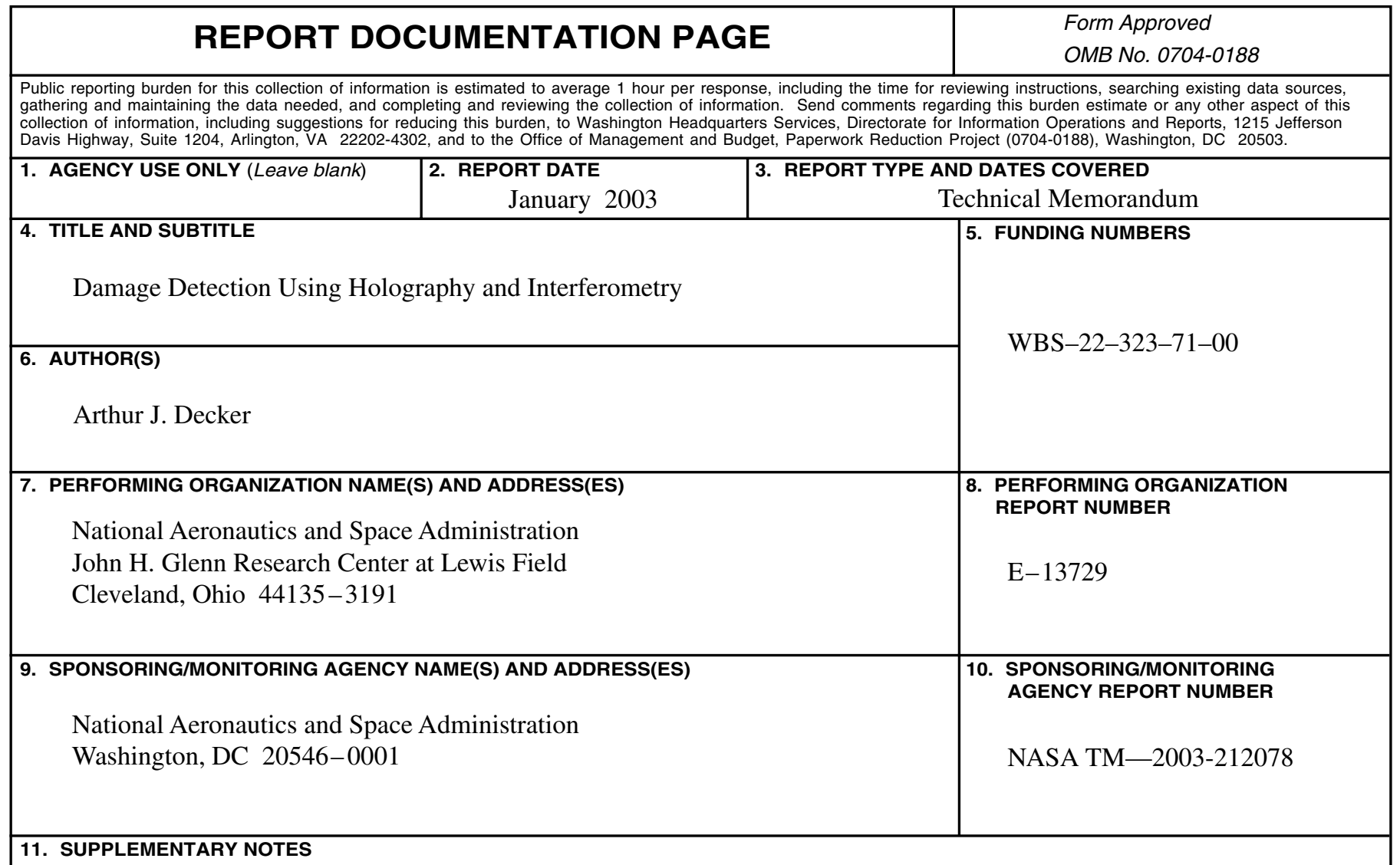

Responsible person, Arthur J. Decker, organization code 5520, 216-433-3639.

12a. DISTRIBUTION/AVAILABILITY STATEMENT

12b. DISTRIBUTION CODE

Unclassified - Unlimited

Subject Category: 35

Distribution: Nonstandard

Available electronically at http://gltrs.grc.nasa.gov

This publication is available from the NASA Center for AeroSpace Information, 301-621-0390.

13. ABSTRACT (Maximum 200 words)

This paper reviews classical approaches to damage detection using laser holography and interferometry. The paper then details the modern uses of electronic holography and neural-net-processed characteristic patterns to detect structural damage. The design of the neural networks and the preparation of the training sets are discussed. The use of a technique to optimize the training sets, called folding, is explained. Then a training procedure is detailed that uses the holographymeasured vibration modes of the undamaged structures to impart damage-detection sensitivity to the neural networks. The inspections of an optical strain gauge mounting plate and an International Space Station cold plate are presented as examples.

14. SUBJECT TERMS

Holography; Interferometry; Non-destructive evaluation; Neural network
15. NUMBER OF PAGES

33

16. PRICE CODE

\begin{tabular}{|c|c|c|}
\hline $\begin{array}{c}\text { 17. SECURITY CLASSIFICATION } \\
\text { OF REPORT } \\
\text { Unclassified }\end{array}$ & $\begin{array}{c}\text { 18. SECURITY CLASSIFICATION } \\
\text { OF THIS PAGE } \\
\text { Unclassified }\end{array}$ & $\begin{array}{c}\text { 19. SECURITY CLASSIFICATION } \\
\text { OF ABSTRACT } \\
\text { Unclassified }\end{array}$ \\
\hline
\end{tabular}

NSN 7540-01-280-5500

Standard Form 298 (Rev. 2-89)

Prescribed by ANSI Std. Z39-18 298-102 

